\title{
Genome-wide CNV analysis replicates the association between GSTM1 deletion and bladder cancer: a support for using continuous measurement from SNP-array data
}

Gaëlle Marenne ${ }^{1,2}$, Francisco X Real 1,3, Nathaniel Rothman ${ }^{4}$, Benjamin Rodríguez-Santiago 3,5, Luis Pérez-Jurado 3,5,6,7, Manolis Kogevinas ${ }^{8,9,10,11}$, Montse García-Closas ${ }^{4}$, Debra T Silverman ${ }^{4}$, Stephen J Chanock ${ }^{4 \dagger}$,

Emmanuelle Génin ${ }^{2 \dagger}$ and Núria Malats ${ }^{1 *+}$

\begin{abstract}
Background: Structural variations such as copy number variants (CNV) influence the expression of different phenotypic traits. Algorithms to identify CNVs through SNP-array platforms are available. The ability to evaluate well-characterized CNVs such as GSTM1 (1p13.3) deletion provides an important opportunity to assess their performance.

Results: 773 cases and 759 controls from the SBC/EPICURO Study were genotyped in the GSTM1 region using TaqMan, Multiplex Ligation-dependent Probe Amplification (MLPA), and Illumina Infinium 1 M SNP-array platforms. CNV callings provided by TaqMan and MLPA were highly concordant and replicated the association between GSTM1 and bladder cancer. This was not the case when CNVs were called using Illumina $1 \mathrm{M}$ data through available algorithms since no deletion was detected across the study samples. In contrast, when the Log R Ratio (LRR) was used as a continuous measure for the 5 probes contained in this locus, we were able to detect their association with bladder cancer using simple regression models or more sophisticated methods such as the ones implemented in the CNVtools package.
\end{abstract}

Conclusions: This study highlights an important limitation in the CNV calling from SNP-array data in regions of common aberrations and suggests that there may be added advantage for using LRR as a continuous measure in association tests rather than relying on calling algorithms.

Keywords: Bladder cancer risk, Glutathione S-transferase mu 1 (GSTM1), Copy number variation (CNV), SNP-array

\section{Background}

The glutathione $S$-transferase mu 1 (GSTM1) gene is located in the 1p13.3 band and codes for the cytosolic enzyme GST- $\mu$ that plays a role in carcinogen detoxification. Many structural variations have been described that overlap this gene in the Database of Genomic Variation [1] (Figure 1). A common copy number variant (CNV) has been well characterized and reported on the basis of the frequency of homozygous deletions of the entire

\footnotetext{
*Correspondence: nmalats@cnio.es

${ }^{\dagger}$ Equal contributors

'Spanish National Cancer Research Center (CNIO), Madrid E-28029, Spain

Full list of author information is available at the end of the article
}

coding region (known as the GSTM1-null genotype), which varies between $29 \%$ and $51 \%$ across ethnic groups [2-4].

Due to the established role of GSTM1 in detoxification and the high frequency of homozygous deletions in the population, GSTM1 has been extensively investigated in association with many chronic diseases, in particular, with asthma [5] and different types of cancers [4,6], including bladder cancer [7]. In a large case-control study, we reported that $63 \%$ of bladder cancer cases and $47 \%$ of controls harbored the GSTM1-null genotype, leading to an $\mathrm{OR}=1.7(95 \% \mathrm{CI} 1.4-2.0)[8]$. This is a well- 


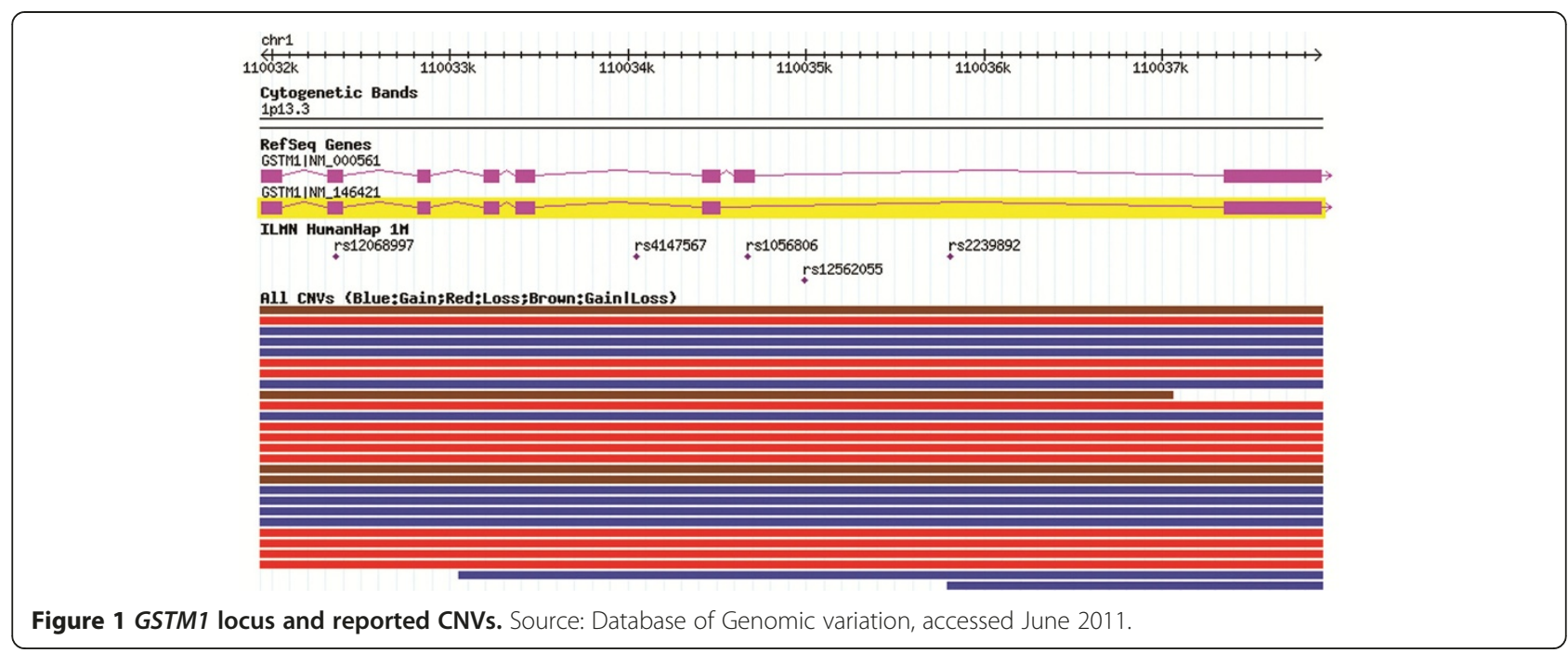

established association as it was replicated in several independent studies and confirmed in a meta-analysis [8].

Reliable and accurate technologies such as qPCR or Multiplex Ligation-dependent Probe Amplification (MLPA) are available to genotype CNVs in targeted regions of the genome. High-throughput SNP-array platforms now offer the possibility to explore CNVs at a genome-wide scale. For instance, Illumina Infinium $1 \mathrm{M}$ provides intensity data of both alleles at each SNP allowing the detection of CNV breakpoints and the estimation of the associated number of copies. Monomorphic probes were also included in genomic regions known to harbor CNVs but that were not well covered by SNPs. Overall, this platform contains 1,071,820 probes, among them 206,665 are located in reported CNV regions and 17,202 are monomorphic probes specially designed for CNV purpose.

Assessing $\mathrm{CNV}$ data across the genome-wide level using SNP-arrays is a daunting problem and requires stringent quality control (QC) measures to minimize the noise related to an analytical scheme that relies on sliding windows across SNPs [9-11]. The assessment also requires that CNVs is called based on the raw intensity data (Log-R ratio, LRR) from each probe. Several calling algorithms, such as PennCNV, have been developed $[9,10,12,13]$ but these algorithms have a very low sensitivity when applied to large data sets [14-16]. In turn, the probability of false negatives remains a major challenge.

To limit false negative callings, a strategy that bypasses the calling step and directly performs the association test using LRR measures has been proposed [17-19]. Alternatively, methods have been developed allowing the simultaneous calling and association test estimation. These methods account better for the calling uncertainties but are yet to be validated in sufficiently large studies [20,21].
The objective of this study was to compare the assessment of a CNV at the GSTM1 locus and its association with bladder cancer by applying LRR and PenCNV to data derived from the Illumina Infinium $1 \mathrm{M}$ SNP-array platform with that derived from TaqMan (qPCR) and MLPA genotyping in subjects included in the Spanish Bladder Cancer/EPICURO (SBC/EPICURO) Study.

\section{Methods \\ Samples}

The SBC/EPICURO Study is a hospital-based case-control study conducted between 1998 and 2001 and described in detail elsewhere [8]. In summary, 18 hospitals in 5 Spanish regions (Barcelona, Vallès/Bages, Alicante, Asturias, Tenerife) participated in the Study with a total of 1,219 cases and 1,271 controls having been interviewed. Controls were matched to cases for gender, age and hospital. Detailed epidemiological information of known and potential risk factors for bladder cancer was collected. Genomic DNA was available for most of the individuals; and after exclusion based on DNA quality, tumor morphology and ethnicity [8], a total of 2,314 individuals (1,157 cases and 1,157 controls) were suitable for the genetic analysis.

Informed consent from all subjects and ethical approval from local and NCI, USA, institutional review boards were obtained.

\section{GSTM1 genotyping}

Three genotyping methods were applied to assess the number of copies at the GSTM1 locus. TaqMan assays were conducted at the Genotyping Core Facility - National Cancer Institute (CGF-NCI), USA, using the SNP500CancerID: GSTM1-02 probe [8]. MLPA assays were performed at the Pompeu Fabra University (UPF, Barcelona) by using optimized custom probes described 
elsewhere [22]. A genome-wide Illumina Infinium $1 \mathrm{M}$ SNP-array scan was performed at the CGF-NCI, USA [7]. The latter platform provided information on 5 probes located in the the GSTM1 locus (Figure 1).

While a different number of individuals were analyzed by each platform, a common set of 1,532 blood-derived samples (773 cases and 759 controls) was available for comparison. Details on the sample sets used for each genotyping platforms are provided in Additional file 1 Table S1 and Additional file 1 Figure S1.

\section{Retrieving CNV information from Illumina Infinium $1 \mathrm{M}$ SNP-array}

The Beadstudio software (Illumina Inc.) was used to process the data. Briefly, for each SNP probe, allele specific fluorescence intensities corresponding to the two alleles, named respectively $\mathrm{A}$ and $\mathrm{B}$, were obtained and normalized to adjust for global differences in intensity and to scale the data as described in [23]. Genotype clusters have been calculated using our own data. The reliability of the SNP genotyping was evaluated through the analysis of duplicated samples; the observed concordance was $>99.5 \%$. The log R Ratio (LRR) was computed by taking the $\log 2$ value of the ratio of the sum of the normalized intensities, $R_{\text {obs }}$, divided the value $R_{\exp }$ expected based on the genotype clusters. The LRR value depends on the number of CNV copies carried by the individual: typically for individuals belonging to the genotype clusters who carry only 2 copies (normal state), the LRR is around 0 , while for individuals carrying less or more copies, the LRR is expected to be negative and positive, respectively. Beadstudio also computes the proportion of $\mathrm{B}$ alleles in the genotype, referred to as the $\mathrm{B}$ allele frequency or BAF, from the normalized intensities. For individuals carrying two copies, the BAF should be around $0,0.5$ or 1 , depending on whether their genotypes are $\mathrm{AA}, \mathrm{AB}$, or $\mathrm{BB}$, respectively.

For CNV calling, we used PennCNV [9] as, for our data set we obtained a better reliability $(0.65)$ based on replicated samples for this algorithm in comparison to other two algorithms [16]. PennCNV implements a HiddenMarkov model (HMM) in which the hidden states are the number of copies (from 0 to 4 copies), and the observed states are the LRR and the BAF values at each probe. One of the HMM parameters is the vector of expected LRR values for each hidden state, the default values for $0,1,2,3$ and 4 copies are respectively -3.53 , $-0.66,0,0.40$ and 0.68 . According to PennCNV authors recommendations, we excluded all individual samples fulfilling at least one of the following criteria: a standard deviation of the LRR values over the $1 \mathrm{M}$ probes $>0.28$, a median BAF value out of the range [0.45 - 0.55], a BAF drift $>0.002$, a wave factor out of $[-0.04-0.04]$. The BAF drift summarizes the departure of the BAF from the expected values when 2 copies ( $0,0.5$ and 1$)$. The wave factor aims to identify samples in which the LRR is not consistent across the genome; it summarizes the variability of the average LRR values in sliding windows.

\section{Statistical method for association testing with LRR}

The association between GSTM1 signal and bladder cancer risk was first tested using the LRR values. To this end, we used logistic regression models where, after adjustment for gender, age, region and tobacco consumption, the disease status was modeled as a function of the LRR at each of the five probes located in the gene (5 tests were performed). Second, we applied the association testing method implemented in the CNVtools package, which unifies genotyping and association testing into a single model by incorporating a dichotomous disease variable into the mixture model for the signal [20]. As recommended by the authors, the method was run using a summarized measure of the LRR across the 5 SNPs located in the GSTM1 gene obtained after applying a principal component analysis and followed by a linear discriminant function. This analysis was also adjusted for gender, region, tobacco consumption and age. Since CNVtools only allows the adjustment for qualitative variables, we categorized the age in 4 classes according to its quartiles.

Analyses were performed using the statistical software R2.9. (http://www.r-project.org) and the Vennarable $R$ package (Jonathan Swinton).

\section{Results}

The concordance rate between TaqMan and MLPA for GSTM1-null identification was high but not complete: 96.2\% over the entire sample (cases and controls) and 95.8\% when considering only the controls. TaqMan and MLPA detected 402 (52.96\%) and 401 (52.83\%) controls carrying a homozygous deletion, respectively, and 289 controls (38.1\%, both platforms) carrying a heterozygous deletion (Additional file Table S2). These values were consistent with the reported rate of deletion in the European population. In contrast, when we conducted an analysis with PennCNV on the Illumina 1M SNP-array data, no deletion was detected among the 759 controls and 773 cases.

As shown in Figure 2, the average LRR values for the 5 Illumina probes located at the GSTM1 locus was higher than expected based on the number of copies reported by TaqMan or MLPA. Indeed, for the individuals with two copies according to both TaqMan and MLPA, the LRR for the five probes was 0.17 on average, higher than 0 , the value expected when 2 copies of the gene are present. Similarly, although negative LRR values are expected when a deletion is present, we observed positive values $(0.08)$ in individuals with one copy and only 


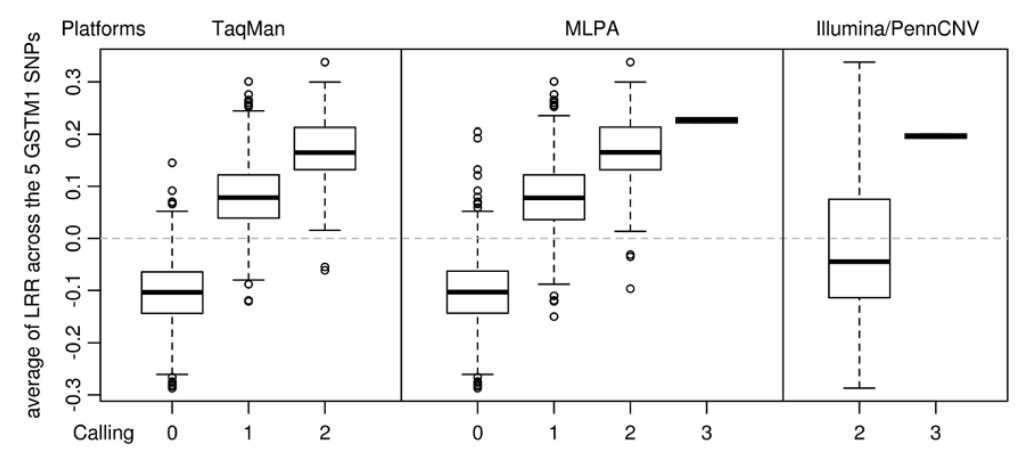

Figure 2 LRR distribution according to CNV callings. Average LRR from the 5 probes located at GSTM1 according to the CNV calling obtained from TaqMan, MLPA and Illumina/PennCNV.

slightly negative values $(-0.10)$ in individuals with a homozygous deletion according to TaqMan and MLPA callings. These differences between the observed pattern of LRR at the GSTM1 locus and the one expected by the PennCNV algorithm could explain that no detection was detected by PennCNV.

However, there was a strong correlation between LRR values and the estimated number of copies using TaqMan or MLPA (analysis of variance - $\mathrm{p}<10^{-15}$ ), suggesting that the LRR values might be useful as an exploratory approach (Figure 2). Using LRR values from each of the five probes as explanatory variables in the logistic regression models, we detected the association with bladder cancer risk for three of them: (rs4147567: $\mathrm{p}=8.00 \times 10^{-4} ; \quad \mathrm{rs} 2239892: \mathrm{p}=0.01$ and rs12068997: $\mathrm{p}=0.02)$ (Table 1). Estimated OR at the 5 SNPs (Table 1) was $<1.0$ indicating that an increase of the LRR was protective for bladder cancer, which was concordant with the evidence in the literature. Indeed, applying the callings provided by TaqMan and MLPA in this sample, we obtained a significant association $\left(\mathrm{p}=3.40 \times 10^{-4}, \mathrm{OR}=\right.$ $0.74 \quad[0.62-0.87]$ and $\mathrm{p}=1.15 \times 10^{-4}, \mathrm{OR}=0.72[0.61$ 0.85 ], respectively) between the trend on the number of copies at GSTM1 and bladder cancer risk.
When using CNVtools to test for association, individuals were clustered into two categories based on their copy numbers. These categories fitted well with their actual status of having a homozygous deletion or not as detected by MLPA or TaqMan. We observed a high concordance rate, $93.7 \%$ and $93.0 \%$, between CNVtools clustering and TaqMan and MLPA callings across the overall sample set. An association signal was detected $(\mathrm{OR}=$ $0.66, \mathrm{p}=1.74 \times 10^{-3}$ ) using this method adjusting for age, gender, region and tobacco use, replicating the known association between GSTM1 and bladder cancer.

\section{Discussion}

Genome structural variants and, particularly $\mathrm{CNV}$, are thought to play an important role in phenotypic variation and in the development of many complex diseases. In the last few years, several calling algorithms have been developed to identify $\mathrm{CNVs}$ at the whole genome scale using the same SNP-chips used to perform GWAS. However, studies that have evaluated the available tools have concluded that they lack sensitivity leading to a large number of false negative callings [1416]. While PennCNV algorithm was found to be the one performing the best in previous comparisons, here

Table 1 Association between GSTM1 and bladder cancer

\begin{tabular}{|c|c|c|c|c|c|c|}
\hline \multirow[b]{2}{*}{ Data } & \multicolumn{2}{|r|}{ Controls } & \multicolumn{2}{|r|}{ Cases } & \multirow[b]{2}{*}{ OR $[95 \% \mathrm{Cl}]$} & \multirow[b]{2}{*}{ p-value } \\
\hline & $\mathrm{N}$ & average (std) of LRR & $\mathrm{N}$ & average (std) of LRR & & \\
\hline rs12068997 $(110,032,359)$ & 756 & $-0.0091(0.1066)$ & 767 & $-0.0223(0.1086)$ & $0.30[0.11-0.82]$ & 0.0190 \\
\hline rs4147567 $(110,034,047)$ & 757 & $-0.0138(0.2810)$ & 767 & $-0.0642(0.2661)$ & $0.51[0.35-0.76]$ & $8.47 \times 10^{-4}$ \\
\hline rs1056806 $(110,034,670)$ & 757 & $-0.0031(0.1105)$ & 767 & $-0.0096(0.1061)$ & $0.67[0.25-1.80]$ & 0.4279 \\
\hline rs12562055 $(110,034,988)$ & 756 & $-0.0001(0.1266)$ & 767 & $-0.0069(0.1244)$ & $0.83[0.36-1.95]$ & 0.6754 \\
\hline rs2239892 $(110,035,809)$ & 757 & $-0.0216(0.2099)$ & 767 & $-0.0474(0.2043)$ & $0.51[0.30-0.85]$ & 0.0103 \\
\hline CNVtools & 756 & & 767 & & 0.66 & $1.74 \times 10^{-3}$ \\
\hline TaqMan & 757 & & 767 & & $0.74[0.62-0.87]$ & $3.40 \times 10^{-4}$ \\
\hline MLPA & 757 & & 767 & & $0.72[0.61-0.85]$ & $1.15 \times 10^{-4}$ \\
\hline
\end{tabular}

The association was performed by logistic regression models adjusted for age, gender, region and smoking status. 
we demonstrate the lack of sensitivity of PennCNV in a particular scenario. In the well-characterized region of GSTM1, we found that PennCNV did not detect any deletion in a large sample of cases with bladder cancer and controls where homozygous deletion was known to have a frequency of $50 \%$ using Taqman and MLPA technologies. Because PennCNV was designed to identify unknown $\mathrm{CNV}$ regions, we also applied the cnvHap algorithm that was designed to genotype known $\mathrm{CNV}$ regions [24]. As expected, cnvHap did not detect any deletion in the GSTM1 region in our sample, either. It is noteworthy the fact that, using $\mathrm{CNV}$ calls derived from Illumina $1 \mathrm{M}$ platform, GSTM1 would have never been associated with bladder cancer. However, when individual probe LRR values are compared between cases and controls, the association can be detected and provide results similar to those obtained when using Taqman or MLPA. This observation clearly shows that PennCNV lacks sensitivity to detect $\mathrm{CNV}$ in the GSTM1 region.

A possible explanation for the lack of sensitivity of PennCNV (and cnvHap) is the high frequency of the GSTM1 deletion in the studied population. Indeed, CNV calling is done using the LRR that depends both on the observed $\left(R_{\text {obs }}\right)$ and the expected $\left(R_{\exp }\right) R$ values. The $R_{\exp }$ is determined based on the clusters of genotypes. In the case of GSTM1 where the homozygous deletion is very frequent, these clusters include a high number of subjects with a homozygous deletion (GSTM1-null genotype). Thus, $R_{\text {obs }}$ and $R_{\text {exp }}$ are expected to be similar in a GSTM1-null individual and, accordingly, the LRR value is around 0 . The normalization process could also play a role as it aims at finding three clusters and this is not possible for GSTM1 locus since the BAF of homozygous deleted sample is uniformly distributed between 0 and 1 , thus normalization is affecting the intensity values, too.

The fact that the association between GSTM1 CNV and bladder cancer can be detected with LRR values without applying a calling CNV confirms the utility of this measure as a complementary screening strategy to test for association at the genome-wide level, as already suggested [17-19]. Indeed, the LRR is a continuous measure that approximates and correlates well with the actual discrete number of copies. Nonetheless, it is affected by the noise contained in the intensity measurement of both alleles obtained through the hybridization experiments. Thus, using LRR in the association test may decrease the power of some probes in detecting the association in comparison of using an accurate calling of the discrete number of copies. This loss of power would explain that two of the five probes located in the GSTM1 locus failed to show association with bladder cancer risk in our study, and that the three significant probes only showed a moderate significant $\mathrm{p}$-values (between $8 \times 10^{-4}$ and 0.019 , Table 1). Nevertheless, even if the significance of these probes was moderate, we observed an excess of significant p-values in comparison to what we could expect under the null hypothesis of no association in that region. Thus, methods working at the genome-wide level and searching for regions with an excess of significant probes could have identified the GSTM1 region in our study. Alternatively, CNVtools, performing a joint calling and association testing, might also be considered, though it is more difficult to apply than that based on LRR and takes longer to run. The main caveat with CNVtools and equivalent methods is the definition of regions of interest.

The GSTM1 deletion is located in a region of high sequence homology neighbored by a segmental duplication and this might explain that its breakpoints may slightly vary and, thus, the difficulties of calling. However, the locus is defined since the deletion in GSTM1 was already known and approaches based in probes are able to identify it. Nevertheless, there might be other still unknown CNVs in the genome showing similar characteristics that might thus not be easy to call [25]. To increase sensitivity in CNV identification at the whole genome scale, we propose performing a genome-wide screen for association using LRR values at each probe and then applying CNVtools for a fine-tuning in the most promising regions.

\section{Conclusion}

In conclusion, our study provides insights into the limitations of $\mathrm{CNV}$-calling algorithms applied to SNP-array platforms in regions harboring common CNVs, especially those with full gene deletions. Though our results focused on a previously characterized CNV, they raise the possibility that there could be a substantial problem across unknown regions of the genome with common CNVs. On the other hand, we showed that by comparing LRR between cases and controls we were able to identify hot genomic areas associated with the trait of interest, supporting the use of this exploratory association assessment at the whole-genome level, which should be pursued with promising efficient calling algorithms.

\section{Additional file}

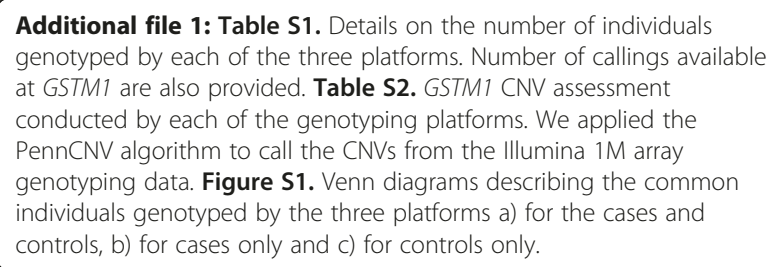

Additional file 1: Table S1. Details on the number of individuals genotyped by each of the three platforms. Number of callings available at GSTM1 are also provided. Table S2. GSTM1 CNV assessment conducted by each of the genotyping platforms. We applied the PennCNV algorithm to call the CNVs from the Illumina $1 \mathrm{M}$ array genotyping data. Figure S1. Venn diagrams describing the common individuals genotyped by the three platforms a) for the cases and controls, b) for cases only and c) for controls only.

\section{Competing interest}

We declare we have no competing interest. 


\section{Authors' contributions}

GM, SJC, NM and EG participated in the design of the study and drafted the manuscript. GM performed the statistical analysis. FXR, NR, BR, LP, MK, MG and DTS are involved in the SBCS study and help to draft the manuscript. All authors read and approved the final manuscript.

\section{Authors' information}

Co-last authors: Núria Malats, Emmanuelle Génin and Stephen J Chanock

\section{Acknowledgements}

We thank Guillermo Pita, Laia Palencia, Kevin Jacobs and Gabriela Andrés, for their technical help; physicians, field-workers and study participants for continuous support during the study conduction.

This work was partially supported by the Fondo de Investigación Sanitaria Spain (G03/174, PI051436, Pl061614, Fl09/00205); Acc.Integrada HispanoFrancesa, Ministerio de Ciencia e Innovación (HF2008-0069), Red Temática de Investigación Cooperativa en Cáncer (RTICC), Instituto de Salud Carlos III, Ministry of Health, Spain; the Intramural Research Program of the Division of Cancer Epidemiology and Genetics, National Cancer Institute, USA; and by the EU (HEALTH-F2-2008-201663); and by Egide-PHRC Picasso travel grant.

\section{Author details}

${ }^{1}$ Spanish National Cancer Research Center (CNIO), Madrid E-28029, Spain. ${ }^{2}$ Inserm UMR-S946, Univ. Paris Diderot, Institut Universitaire d'Hématologie, Paris F-75010, France. 'Departament de Ciències Experimentals i de la Salut, Universitat Pompeu Fabra, Barcelona E-08003, Spain. ${ }^{4}$ Division of Cancer Epidemiology and Genetics, National Cancer Institute, Bethesda, MD 20852-4907, USA. ${ }^{5}$ Centro de Investigación Biomédica en Red de Enfermedades Raras (CIBERER), Barcelona E-08003, Spain. ${ }^{6}$ Programa de Medicina Molecular i Genètica, Hospital Universitari Vall d'Hebron, Barcelona E-08003, Spain. ${ }^{7}$ Department of Genome Sciences, University of Washington, Seattle, WA 98195, USA. ${ }^{8}$ Municipal Institute of Medical Research (IMIM-Hospital del Mar), Barcelona E-08003, Spain. ${ }^{9}$ Centre for Research in Environmental Epidemiology (CREAL), Barcelona E-08003, Spain. ${ }^{10}$ Centro de Investigación Biomédica en Red en Epidemiología y Salud Pública (CIBERESP), Barcelona E-08003, Spain. ${ }^{11}$ National School of Public Health, Athens G-11521, Greece.

Received: 25 November 2011 Accepted: 20 July 2012

Published: 20 July 2012

\section{References}

1. lafrate AJ, Feuk L, Rivera MN, Listewnik ML, Donahoe PK, Qi Y, Scherer SW, Lee C: Detection of large-scale variation in the human genome. Nat Genet 2004, 36:949-951.

2. Cotton SC, Sharp L, Little J, Brockton N: Glutathione S-transferase polymorphisms and colorectal cancer: a HuGE review. Am J Epidemiol 2000, 151:7-32.

3. Engel LS, Taioli E, Pfeiffer R, Garcia-Closas M, Marcus PM, Lan Q, Boffetta P, Vineis P, Autrup H, Bell DA, Branch RA, Brockmoller J, Daly AK, Heckbert SR, Kalina I, Kang D, Katoh T, Lafuente A, Lin HJ, Romkes M, Taylor JA, Rothman N: Pooled analysis and meta-analysis of glutathione S-transferase M1 and bladder cancer: a HuGE review. Am J Epidemiol 2002, 156:95-109.

4. d'Errico A, Malats N, Vineis P, Boffetta P: Review of studies of selected metabolic polymorphisms and cancer. IARC Sci Publ 1999, 148:323-393.

5. Minelli C, Granell R, Newson R, Rose-Zerilli MJ, Torrent M, Ring SM, Holloway JW, Shaheen SO, Henderson JA: Glutathione-S-transferase genes and asthma phenotypes: a Human Genome Epidemiology (HuGE) systematic review and meta-analysis including unpublished data. Int J Epidemio/ 2010, 39:539-562.

6. Parl FF: Glutathione S-transferase genotypes and cancer risk. Cancer Lett 2005, 221:123-129.

7. Rothman N, Garcia-Closas M, Chatterjee N, Malats N, Wu X, Figueroa JD, Real FX, Van Den Berg D, Matullo G, Baris D, Thun M, Kiemeney LA, Vineis P, De Vivo I, Albanes D, Purdue MP, Rafnar T, Hildebrandt MA, Kiltie AE, Cussenot O, Golka K, Kumar R, Taylor JA, Mayordomo Jl, Jacobs KB, Kogevinas M, Hutchinson A, Wang Z, Fu YP, Prokunina-Olsson L, Burdett L, Yeager M, Wheeler W, Tardon A, Serra C, Carrato A, Garcia-Closas R, Lloreta J, Johnson A, Schwenn M, Karagas MR, Schned A, Andriole G Jr, Grubb R 3rd, Black A, Jacobs EJ, Diver WR, Gapstur SM, Weinstein SJ, Virtamo J, Cortessis VK, Gago-Dominguez M, Pike MC, Stern MC, Yuan JM, Hunter DJ,
McGrath M, Dinney CP, Czerniak B, Chen B, Yang H, Vermeulen SH, Aben KK, Witjes JA, Makkinje RR, Sulem P, Besenbacher S, Stefansson K, Riboli E, Brennan P, Panico S, Navarro C, Allen NE, Bueno-de-Mesquita HB, Trichopoulos D, Caporaso N, Landi MT, Canzian F, Ljungberg B, Tjonneland A, Clavel-Chapelon F, Bishop DT, Teo MT, Knowles MA, Guarrera S, Polidoro S, Ricceri F, Sacerdote C, Allione A, Cancel-Tassin G, Selinski S, Dietrich H, Fletcher T, Rudnai P, Gurzau E, Koppova K, Bolick SC, Godfrey A, Xu Z, SanzVelez II, M DG-P, Sanchez M, Valdivia G, Porru S, Benhamou S, Hoover RN, Fraumeni JF Jr, Silverman DT, Chanock SJ: A multi-stage genome-wide association study of bladder cancer identifies multiple susceptibility loci. Nat Genet 2010, 42:978-984.

8. Garcia-Closas M, Malats N, Silverman D, Dosemeci M, Kogevinas M, Hein DW, Tardon A, Serra C, Carrato A, Garcia-Closas R, Lloreta J, Castano-Vinyals G, Yeager M, Welch R, Chanock S, Chatterjee N, Wacholder S, Samanic C, Tora M, Fernandez F, Real FX, Rothman N: NAT2 slow acetylation, GSTM1 null genotype, and risk of bladder cancer: Results from the Spanish Bladder Cancer Study and meta-analyses. Lancet 2005, 366:649-659.

9. Wang K, Li M, Hadley D, Liu R, Glessner J, Grant SF, Hakonarson H, Bucan M: PennCNV: an integrated hidden Markov model designed for highresolution copy number variation detection in whole-genome SNP genotyping data. Genome Res 2007, 17:1665-1674.

10. Colella S, Yau C, Taylor JM, Mirza G, Butler H, Clouston P, Bassett AS, Seller A, Holmes CC, Ragoussis J: QuantiSNP: an Objective Bayes Hidden-Markov Model to detect and accurately map copy number variation using SNP genotyping data. Nucleic Acids Res 2007, 35:2013-2025.

11. Itsara A, Cooper GM, Baker C, Girirajan S, Li J, Absher D, Krauss RM, Myers RM, Ridker PM, Chasman DI, Mefford H, Ying P, Nickerson DA, Eichler EE: Population analysis of large copy number variants and hotspots of human genetic disease. Am J Hum Genet 2009, 84:148-161.

12. Korn JM, Kuruvilla FG, McCarroll SA, Wysoker A, Nemesh J, Cawley S, Hubbell E, Veitch J, Collins PJ, Darvishi K, Lee C, Nizzari MM, Gabriel SB, Purcell S, Daly MJ, Altshuler D: Integrated genotype calling and association analysis of SNPs, common copy number polymorphisms and rare CNVs. Nat Genet 2008, 40:1253-1260

13. Pique-Regi R, Caceres A, Gonzalez JR: R-Gada: a fast and flexible pipeline for copy number analysis in association studies. BMC Bioinforma 2010, 11:380.

14. Winchester L, Yau C, Ragoussis J: Comparing CNV detection methods for SNP arrays. Brief Funct Genomic Proteomic 2009, 8:353-366.

15. Dellinger AE, Saw SM, Goh LK, Seielstad M, Young TL, Li YJ: Comparative analyses of seven algorithms for copy number variant identification from single nucleotide polymorphism arrays. Nucleic Acids Res 2010, 38:e105.

16. Marenne G, Rodriguez-Santiago B, Closas MG, Perez-Jurado L, Rothman N, Rico D, Pita G, Pisano DG, Kogevinas M, Silverman DT, Valencia A, Real FX, Chanock SJ, Genin E, Malats N: Assessment of copy number variation using the Illumina Infinium 1 M SNP-array: a comparison of methodological approaches in the Spanish Bladder Cancer/EPICURO study. Hum Mutat 2011, 32:240-248.

17. McCarroll SA, Altshuler DM: Copy-number variation and association studies of human disease. Nat Genet 2007, 39:S37-S42.

18. Stranger BE, Forrest MS, Dunning M, Ingle CE, Beazley C, Thorne N, Redon R, Bird CP, de Grassi A, Lee C, Tyler-Smith C, Carter N, Scherer SW, Tavare S, Deloukas P, Hurles ME, Dermitzakis ET: Relative impact of nucleotide and copy number variation on gene expression phenotypes. Science 2007, 315:848-853.

19. Ionita-Laza I, Perry GH, Raby BA, Klanderman B, Lee C, Laird NM, Weiss ST, Lange C: On the analysis of copy-number variations in genome-wide association studies: a translation of the family-based association test. Genet Epidemiol 2008, 32:273-284.

20. Barnes C, Plagnol V, Fitzgerald T, Redon R, Marchini J, Clayton D, Hurles ME: A robust statistical method for case-control association testing with copy number variation. Nat Genet 2008, 40:1245-1252.

21. Gonzalez JR, Subirana I, Escaramis G, Peraza S, Caceres A, Estivill X Armengol L: Accounting for uncertainty when assessing association between copy number and disease: a latent class model. BMC Bioinforma 2009, 10:172.

22. Rodriguez-Santiago B, Brunet A, Sobrino B, Serra-Juhe C, Flores R, Armengol L, Vilella E, Gabau E, Guitart M, Guillamat R, Martorell L, Valero J, Gutierrez-Zotes A, Labad A, Carracedo A, Estivill X, Perez-Jurado LA: Association of common copy number variants at the glutathione $\mathrm{S}$-transferase genes and rare novel genomic changes with schizophrenia. Mol Psychiatry 2009, 15:1023-1033 
23. Staaf J, Vallon-Christersson J, Lindgren D, Juliusson G, Rosenquist $R$, Hoglund M, Borg A, Ringner M: Normalization of Illumina Infinium wholegenome SNP data improves copy number estimates and allelic intensity ratios. BMC Bioinforma 2008, 9:409.

24. Coin LJ, Asher JE, Walters RG, Moustafa JS, de Smith AJ, Sladek R, Balding DJ, Froguel P, Blakemore Al: cnvHap: an integrative population and haplotypebased multiplatform model of SNPs and CNVs. Nat Methods 2010, 7:541-546.

25. Huang RS, Chen P, Wisel S, Duan S, Zhang W, Cook EH, Das S, Cox NJ,

Dolan ME: Population-specific GSTM1 copy number variation. Hum Mol Genet 2009, 18:366-372.

doi:10.1186/1471-2164-13-326

Cite this article as: Marenne et al:: Genome-wide CNV analysis replicates the association between GSTM1 deletion and bladder cancer: a support for using continuous measurement from SNP-array data. BMC Genomics 2012 13:326.

\section{Submit your next manuscript to BioMed Central and take full advantage of:}

- Convenient online submission

- Thorough peer review

- No space constraints or color figure charges

- Immediate publication on acceptance

- Inclusion in PubMed, CAS, Scopus and Google Scholar

- Research which is freely available for redistribution 